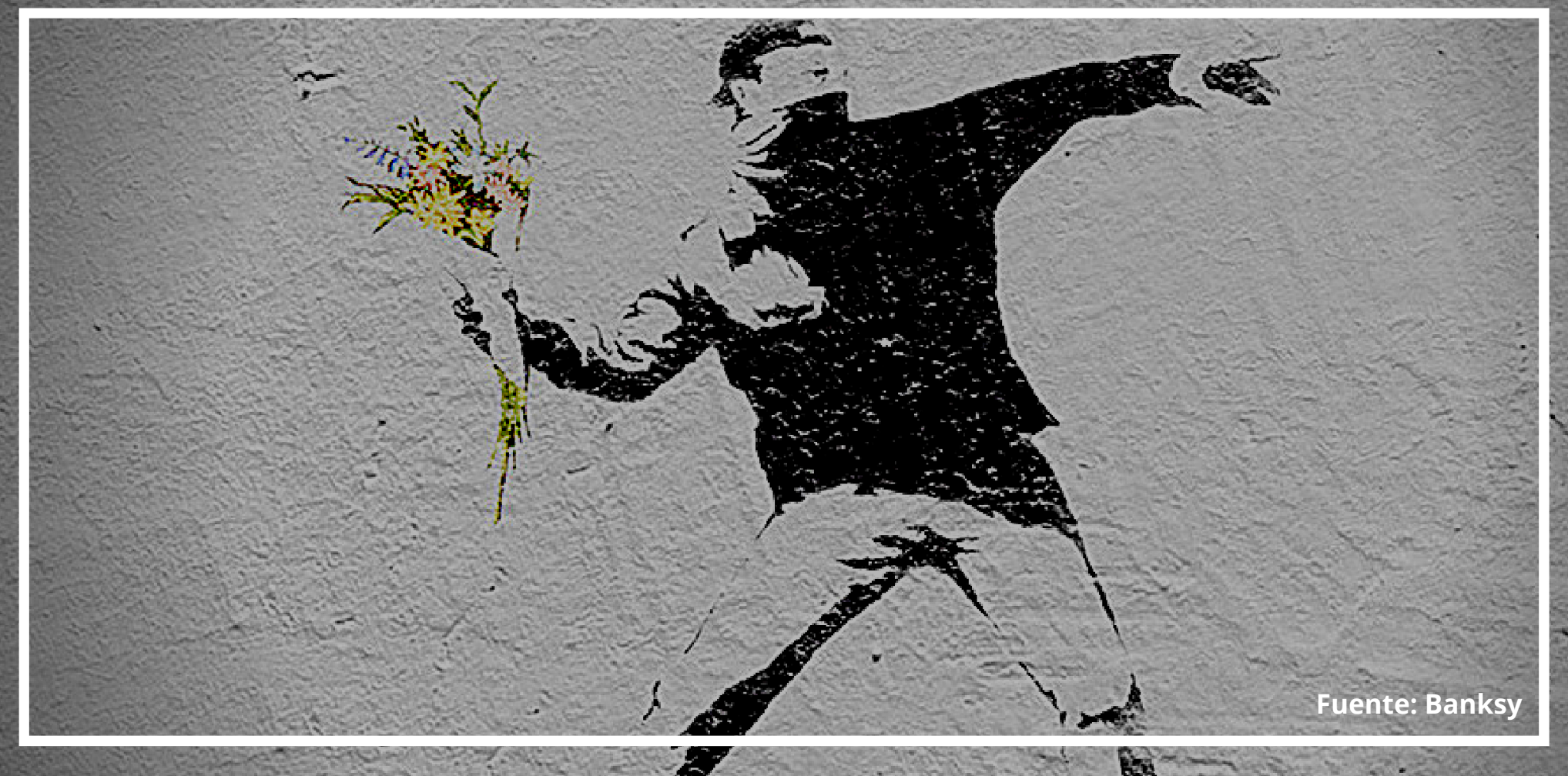

\title{
Podemos hacer del mundo posterior al coronavirus un lugar mucho menos violento
}

doi: $10.52749 /$ rh.v1i1.1

\section{ROBERT MUGGAH}

Director del Grupo SecDev, cofundador del Instituto Igarapé y autor, junto con Ian Goldin, de Terra Incognita: 100 mapas para sobrevivir a los próximos 100 años.

$\checkmark$ muggah@hei.unige.ch

\section{STEVEN PINKER IiD https://orcid.org/0000-0002-2319-4085}

Johnstone Family Professor en el Departamento de Psicología de la Universidad de Harvard y autor de 12 libros, el más recientemente se titula Enlightenment Now: The Case for Reason, Science, Humanism, and Progress.

$\checkmark$ pinker@wjh.harvard.edu 円ttps://stevenpinker.com/

Resumen. La pandemia ha disminuido algunos tipos de delitos y ha aumentado otros. Pero el mundo es mucho más seguro de lo que solía ser, y sabemos cómo hacerlo aún más seguro.

Palabras clave: coronavirus, pandemia, violencia

\section{Cómo citar este artículo:}

Muggah, R. \& Pinker, S. (2021). Podemos hacer del mundo posterior al coronavirus un lugar mucho menos violento. (P. Gayozzo, Trad.) Revista Humanista, 1(1), 7-11. https://doi.org/10.52749/rh.vlil.1. (Trabajo original publicado en 2020).

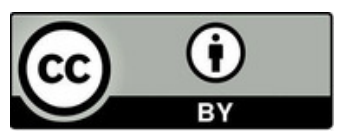




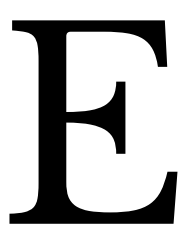

1 mundo está convulsionado por el nuevo coronavirus, pero ese no es el único patógeno que nos aflige. La violencia criminal también es endémica, contagiosa y muy virulenta. Más de 464 000 personas murieron en homicidios en 2017 (el último año del que tenemos datos fiables), al menos cinco veces más de los que mueren en guerra (United Nations, 2019). Millones más sufren lesiones físicas y psicológicas provocadas por el abuso doméstico, las peleas de pandillas y la violencia extrajudicial.

La pandemia de coronavirus seguramente afectará los patrones de esta violencia, pero ¿cómo? La sabiduría convencional sugiere que los momentos de gran estrés producen más violencia, pero los datos no lo confirman: durante la terrible pandemia de gripe española de 1918-1919, ni Estados Unidos ni Gran Bretaña experimentaron un aumento significativo de la violencia. De hecho, las tasas de homicidio cayeron durante la Gran Depresión de la década de 1930. También disminuyeron durante la recesión que comenzó en 2007.

Con los toques de queda nocturnos y las cuarentenas impuestas que mantienen a las personas fuera de las calles y de los bares, algunas formas de delitos violentos están disminuyendo rápidamente en América del Norte (Axelrod, 2020) y partes de América Latina (Brito, 2020). Pero con la gente encerrada y agitada, el abuso doméstico parece estar aumentando (Fielding, 2020). El ciberdelito también está aumentando (Strasburg \& Hinshaw, 2020). Y en países como México que están acosados por el crimen organizado, las tasas de homicidio se han disparado a niveles récord, lo que sugiere un colapso del orden público a medida que se propaga la pandemia (Agren, 2020).

Las perspectivas a más largo plazo son aún menos claras. Una gran preocupación es qué sucederá si las cadenas de suministro se rompen y los precios de los alimentos suben. El precio de los productos alimenticios básicos es un problema de vida o muerte para más del 60 por ciento de la población mundial que depende de la economía informal. También hay temores de un aumento del desorden social cuando los gobiernos hacen cumplir violentamente cierres y cuarentenas, como lo están haciendo en Kenia, Sudáfrica y Uganda
(Ratcliffe, 2020). En Filipinas, el presidente ha emitido órdenes de disparar a matar para quienes protestan por el cierre (Amnesty International, 2020). Mientras tanto, en Brasil, El Salvador e Italia, las pandillas y los grupos mafiosos están imponiendo sus propios toques de queda para evitar que el virus se propague (Betz, 2020; Schipani \& Harris, 2020; WilliamsAnnunziata, 2020).

Pero la posibilidad de que ciertas formas de violencia se intensifiquen a raíz de la pandemia no debería cegarnos al hecho de que el mundo, en promedio, se ha convertido en un lugar mucho más seguro. La mayoría de los países experimentaron una fuerte disminución de las tasas de violencia letal durante las últimas dos décadas. Y lo que es igualmente importante, estamos empezando a comprender, con base en la evidencia de todo el mundo, qué tipos de políticas y programas funcionan realmente para disminuir los delitos violentos y cuáles no. Armados con esta evidencia, tenemos el poder de reducir aún más la violencia, ya sea causada por la pandemia o no.

La escala de estas reducciones en la violencia letal ha sido asombrosa. Entre 1990 y 2015, América del Norte redujo a la mitad su tasa de homicidios, que ahora está cerca de mínimos históricos en Estados Unidos y Canadá. Los países europeos también registraron fuertes caídas. En Asia, la tasa de homicidios fue un 38 por ciento más baja en 2017 que en el año 2000 . Y descensos de más del 50 por ciento se han registrado en países como Colombia, Ecuador, Estonia, Kazajstán, Rusia y Sri Lanka.

En muchas ciudades y barrios, la caída de los homicidios ha sido asombrosa. En los Estados Unidos, la mayoría de las 30 ciudades más grandes son mucho más seguras hoy que hace algunas décadas (Lau, 2019). Nueva York registró más de 2.200 asesinatos en 1990, pero apenas 300 en 2019. Washington, D.C., experimentó una caída de más de 700 homicidios en 1990 a solo 163 en 2019. Otras ciudades de América del Norte y Europa Occidental han seguido una tendencia similar (Renno Santos \& Testa, 2019).

Las buenas noticias incluso provienen de algunos de los lugares más violentos del mundo. La antigua capital de los asesinatos del planeta, Medellín, Colombia, experimentó un vertiginoso descenso de la violencia letal, de 266 homicidios por cada 100.000 habitantes a prin- 
cipios de la década de 1990 a 30 por cada 100.000 en 2015, una caída de casi el 90 por ciento (Muggah, 2017). La tasa de homicidios de Bogotá cayó de 81 por 100.000 en 1993 a aproximadamente 17 por 100.000 en 2015. São Paulo, una ciudad notoriamente homicida, registra actualmente la tasa de homicidios más baja desde que comenzaron los registros (Bocchini, 2020).

A pesar de estas mejoras, las tasas de homicidio siguen siendo obstinadamente altas en América Latina y el Caribe. La región todavía registra un tercio de los homicidios del mundo, a pesar de que contiene menos del 9 por ciento de la población mundial (Luhnow, 2018). De las 50 ciudades más asesinas del mundo en 2016, 43 estaban en América Latina y el Caribe (The Economist, 2017).

¿Por qué la vida en tantos lugares se ha vuelto mucho más segura? Como sucede tan a menudo, hay muchas causas y no es fácil separarlas con confianza. La violencia individual es en gran parte un juego de hombres jóvenes, y las sociedades con una mayor proporción de adolescentes y adultos jóvenes tienden a tener niveles más altos de delitos violentos. Por el contrario, los países con poblaciones mayores como Japón, Italia y Alemania suelen ser más pacíficos (Brooks et al., 2019).

Pero la distribución por edades de un país cambia lentamente $y$, por sí sola, no puede explicar la reducción de la violencia a la mitad en una década. Cuando comparamos regiones, la violencia se correlaciona estadísticamente con el nivel de desigualdad, tal vez porque los hombres que se encuentran en la parte inferior de una distribución de ingresos muy desigual se vuelven más sensibles al estatus social y reaccionan violentamente a agravios menores. Pero esa hipótesis no puede explicar por qué la violencia está pobremente correlacionada con la desigualdad a lo largo del tiempo: la violencia ha disminuido drásticamente incluso cuando la desigualdad de ingresos ha aumentado en muchos países.

Puede que no sea la desigualdad en los iningresos lo que predice la violencia, sino la desigualdad en la protección contra la violencia por parte de las instituciones. En A Savage Order, la experta en seguridad Rachel Kleinfeld observa que en las sociedades más violentas, el estado actúa como una fuerza de seguridad para las élites más que como un garante universal de la paz. Cuando las sociedades comienzan a confiar su protección en una fuerza policial y un sistema de justicia capaces, llegan a disfrutar de más ley y orden para todos.

Un hilo común en las regiones que han reducido la violencia es la presencia de fuerzas policiales más grandes y mejor capacitadas destinadas a reducir la violencia en los lugares donde esta es peor. La solidaridad social y política son partes esenciales de este círculo virtuoso. Cuando los líderes políticos reclutan a la policía y a las comunidades como socios para hacer cumplir las normas que sancionan el comportamiento delictivo y promueven la seguridad colectiva, los crímenes violentos disminuyen.

El círculo virtuoso de la reducción del crimen a menudo comienza cuando las ciudades y regiones establecen objetivos claros que abarcan múltiples ciclos electorales $y$ feudos administrativos. Los líderes políticos deben decidir que la reducción de los homicidios es una meta alcanzable, no un eslogan de campaña o una fuente de gasto inútil, y destinar los recursos adecuados a la tarea. La implementación de estos planes requiere la participación sostenida de alcaldes, jefes de policía y líderes cívicos y empresariales.

Las políticas y programas deben seleccionarse sobre la base de datos confiables que demuestren que funcionan, en lugar de modas, consignas o esperanzas utópicas de extirpar causas fundamentales como la pobreza y el racismo. La policía debe ser vista no como antagonistas sino como proveedores de servicios que brindan lo que todos en la comunidad quieren: calles y hogares más seguros. Y debido a que la violencia criminal está tan concentrada - sabemos por los datos que una pequeña cantidad de vecindarios y perpetradores representan una gran parte de la violencia - la reducción exitosa del crimen debe enfocar los recursos en los lugares que son más violentos.

Estos esfuerzos pueden basarse en un creciente cuerpo de evidencia sobre lo que funciona y lo que no. En su libro Bleeding Out, que revisa una literatura de miles de estudios sobre la reducción de la violencia, el investigador de justicia penal Thomas Abt muestra que una de las tácticas más efectivas es la vigilancia policial en puntos críticos, que se 
ubica en las ciudades, vecindarios y esquinas de las calles. donde la violencia es más desenfrenada. Una estrategia complementaria probada es la disuasión focalizada, que identifica a las pandillas e individuos más agresivos y les envía el mensaje claro de que serán castigados por cometer actos de violencia y los recompensa (con trabajos, capacitación y otras oportunidades) por abstenerse de hacerlo.

Cuando se identifican posibles alborotadores, otra estrategia cuya eficacia se desprende claramente de la evidencia es la terapia cognitivo-conductual. Estas intervenciones están diseñadas para anular los hábitos de pensamiento desadaptativos y el comportamiento impulsivo que causan la delincuencia criminal, y para enseñar estrategias de autocontrol que pueden detener una escalada de agresión antes de que comience. Incluyen capacitación en el manejo de la ira y habilidades sociales, junto con asesoramiento en estrategias que están diseñadas explícitamente para prevenir la reincidencia.

Estos buenos hábitos pueden reforzarse diseñando entornos urbanos con menos tentaciones a las que resistir, con cierres de bares más tempranos y menos calles oscuras, esquinas apartadas y edificios abandonados. Según el sociólogo Patrick Sharkey, los esfuerzos de renovación urbana y movilización local para recuperar parques, manzanas y plazas abiertas en los Estados Unidos jugaron un papel fundamental en la reducción del crimen y la victimización.

Tan importante como saber qué funciona es saber qué no. Vigilancia policial agresiva y de tolerancia cero, sentencias obligatorias de tres strikes y estás fuera, programas de concientización sobre drogas dirigidos por la policía, las llamadas intervenciones de miedo directo que exponen a niños pequeños a prisiones y presos, recompra de armas de fuego y programas de limpieza de barrios marginales son ineficaces o empeoran las cosas.

El objetivo de evitar que las personas se maten entre sí en grandes cantidades no solo es deseable sino alcanzable. El objetivo de reducir la tasa mundial de homicidios en un 50 por ciento para 2030, aproximadamente un 6,5 por ciento al año, ha sido adoptado por Pathfinders for Peaceful, Just and Inclusive Societies, una coalición de gobiernos, organizaciones nacionales e internacionales, fundaciones $\mathrm{y}$ socios del sector privado.

La primera orden del día es redoblar las intervenciones basadas en evidencia en los países, ciudades y vecindarios más peligrosos, capitalizando nuestro conocimiento de lo que funciona y lo que no, y del hecho de que la violencia letal tiende a concentrarse en pocas áreas y entre un pequeño número de personas. Cuando se aplican las medidas adecuadas en los lugares adecuados, los homicidios y otras formas de delitos violentos pueden disminuir rápidamente.

El valor moral de observar los datos no es solo que sea la única base para elegir políticas que realmente salven vidas. La cuantificación de los objetivos para reducir la violencia también es ética porque trata a todas las vidas como igualmente valiosas. Las acciones para prevenir el mayor número de asesinatos previenen la mayor cantidad de tragedias humanas. Como nos recuerda la pandemia, no hay objetivo más importante que salvar vidas.

\section{Referencias}

Agren, D. (03 de Abril de 2020). Mexico murder rate reaches new high as violence rages amid Covid-19 spread. The Guardian. https://www.theguardian.com/world/2020/apr/03/mexico-murderrate-homicide-coronavirus-covid-19

Amnesty International. (02 de Abril de 2020). Philippines: President Duterte gives "shoot to kill" order amid pandemic response. Amnesty International: https://www.amnesty.org/en/latest/news/2020/04/philippinespresident-duterte-shoot-to-kill-order-pandemic/

Axelrod, T. (03 de Abril de 2020). Crime rates drop across the nation amid coronavirus. The Hill: https://thehill.com/homenews/statewatch/491055-crime-rates-drop-across-the-nation-amidcoronavirus
Betz, B. (08 de Abril de 2020). El Salvador gangs enforce coronavirus lockdown with threats of violence, report says. Fox News: $\quad$ https://www.foxnews.com/world/el-salvador-gangscoronavirus-lockdown

Bocchini, B. (24 de Enero de 2020). São Paulo tem em 2019 a menor taxa de homicídios desde 2001. Agência Brasil: https://agenciabrasil.ebc.com.br/geral/noticia/2020-01/sao-paulotem-em-2019-menor-taxa-de-homicidios-desde-2001

Brito, C. (08 de Abril de 2020). Homicide numbers plummet in Latin American countries during coronavirus quarantines. CBS News: https://www.cbsnews.com/news/coronavirus-latin-america-elsalvador-homicides-covid19/

Brooks, D. J., Brooks, S. G., Greenhill, B. D., \& Haas, M. L. (2019). The Demographic Transition Theory of War: Why Young 
Societies Are Conflict Prone and Old Societies Are the Most Peaceful. International Security, 43(3), 53-95. https://doi.org/10.1162/isec a 00335

Fielding, S. (03 de Abril de 2020). In quarantine with an abuser: surge in domestic violence reports linked to coronavirus. The Guardian. https://www.theguardian.com/usnews/2020/apr/03/coronavirus-quarantine-abuse-domesticviolence

Lau, T. (12 de Junio de 2019). Crime Rates in Largest U.S. Cities Continue to Drop. Brennan Center. https://www.brennancenter.org/our-work/analysis-opinion/crimerates-largest-us-cities-continue-drop

Luhnow, D. (20 de Septiembre de 2018). Latin America Is the Murder Capital of the World. The Wall Street Journal: https://www.wsj.com/articles/400-murders-a-day-the-crisis-oflatin-america-1537455390

Muggah, R. (27 de Noviembre de 2017). Where Are the World's Most Fragile Cities? Bloomber City Lab https://www.bloomberg.com/news/articles/2017-11-27/fixing-theworld-s-most-fragile-cities

Ratcliffe, R. (01 de Abril de 2020). Teargas, beatings and bleach: the most extreme Covid-19 lockdown controls around the world. The Guardian. https://www.theguardian.com/globaldevelopment/2020/apr/01/extreme-coronavirus-lockdowncontrols-raise-fears-for-worlds-poorest

Renno Santos, M., \& Testa, A. (11 de Noviembre de 2019). Homicide is declining around the world because we're getting old. Quartz . https://qz.com/1743595/why-global-homicide-rates-are-declining!
Schipani, A., \& Harris, B. (Marzo de 27 de 2020). Drug gangs in Brazil's favelas enforce coronavirus lockdown. Financial Times. https://www.ft.com/content/aaef1591-2fc5-4e6f-ab840e83b5a146ca

Strasburg, J., \& Hinshaw, D. (24 de Marzo de 2020). Cybercrimina Is Sweep In to Take Advantage of Coronavirus. The Wall Street Journal. https://www.wsj.com/articles/cybercriminals-sweep-in-totake-advantage-of-coronavirus- 11585056466

The Economist. (31 de Marzo de 2017). The world's most dangerous cities. The Economist. https://www.economist.com/graphicdetail/2017/03/31/the-worlds-most-dangerous-cities

United Nations. (8 de Julio de 2019). Homicide kills far more people than armed conflict, says new UNODC study. United Nations Office on Drugs and Crime. https://www.unodc.org/unodc/en/frontpage/2019/July/homicidekills-far-more-people-than-armed-conflict--says-new-unodcstudy.html?ref=fs1

Williams-Annunziata, A. (06 de Abril de 2020). Opinion: In Italy's south, the coronavirus is not the only thing to fear. The Washington Post. https://www.washingtonpost.com/opinions/2020/04/06/italyssouth-coronavirus-is-not-only-thing-fearl

* La Sociedad Secular Humanista del Perú tiene el permiso expreso de Steven Pinker para traducir y republicar el artículo publicado originalmente el 14 de abril del 2020 en el portal del Foreign Policy con el título de "We Can Make the Post-Coronavirus World a Much Less Violent Placer".

Recuperado de: https://foreignpolicy.com/2020/04/14/we-can-make-the-post-coronavirus-world-much-less-violent/

**Artículo traducido por Piero Gayozzo para la Revista Humanista del Fondo Editorial de la Sociedad Secular

Humanista del Perú. Contacto: pgayozzo@ssh.org.pe 